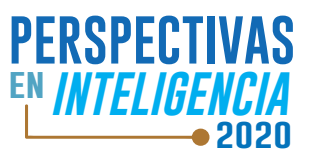

Revista científica en Ciencias Sociales e interdisciplinaria

Bogotá D.C., Colombia

Volumen 12, número 21, enero-diciembre 2020, pp. 203-217

https://doi.org/10.47961/2145194X.232

\title{
Las comunidades indígenas en el constructo de Nación: una revisión teórica
}

The indigenous communities in the construct of Nation: a theoretical review.

\section{Ana María Leguizamón Álvarez ${ }^{{ }^{*}}$ y Wendy Verónica Veloza Mantilla ${ }^{2}$}

(1) Universidad Externado de Colombia, Bogotá D.C. - Colombia, anama.leguizamo@gmail.com

(2) Universidad Externado de Colombia, Bogotá D.C. - Colombia, wendyveloza09@gmail.com

* Autor a quien se dirige la correspondencia

\section{Resumen}

Esta investigación tiene como objetivo aportar, de forma descriptiva, a las razones por las cuales las poblaciones indígenas en América Latina no han apropiado el concepto de identidad nacional. El enfoque de investigación es cualitativo; a través de búsqueda y codificación, por medio de Bibliometrix, se realizó análisis de escritorio con más de 50 artículos indexados, reseñas y capítulos de libros. Los hallazgos muestran que la identidad, la cultura, el pasado común y la naturaleza influyen en la concepción de nación que tienen las poblaciones indígenas. Asimismo, la libre determinación de los pueblos, el escenario internacional y la educación son algunos de los factores que impulsan a las comunidades indígenas latinoamericanas a manifestarse por no sentirse incluidos en sus respectivos Estados. La investigación concluye que las poblaciones indígenas fueron excluidas en la construcción de los Estados-nación en el siglo XIX, de forma que la idea de una sola nación choca con la multiplicidad de identidades y culturas de los pueblos indígenas.

Palabras clave: Comunidades indígenas; identidad cultural; migrante; derechos territoriales; diversidad.

\section{Abstract}

This research has the objective of contributing, in a descriptive way, to the reasons why indigenous populations in Latin America have not appropriated the concept of national identity. The research approach is qualitative; through search and coding, by means of Bibliometrix, desktop analysis was performed with more than 50 indexed articles, reviews and book chapters. The findings show that identity, culture, common past and nature influence the indigenous populations' conception of nation. Likewise, self-determination of peoples, the international scenario and education are some of the factors that drive Latin American indigenous communities to demonstrate because they do not feel included in their respective States. The research concludes that indigenous populations were excluded in the construction of nation-states in the 19th century, so that the idea of a single nation clashes with the multiplicity of identities and cultures of indigenous peoples.

Keywords: Indigenous communities; cultural identity; migrant; territorial rights; diversity. 


\section{Contexto}

Desde finales del siglo XX las poblaciones indígenas han ocupado un papel relevante en la inestabilidad política ${ }^{39}$ de los Estados latinoamericanos. Así, en países de la región, estas poblaciones se han movilizado de forma masiva (Dávalos, 2005) generando presión, y en ocasiones, que los presidentes dimitan de su cargo, tal es el caso de los presidentes de Ecuador: Jamil Mahuad (2000) y Lucio Gutiérrez (2003), así como los presidentes de Bolivia: Gonzalo Sánchez de Lozada (2003) y Carlos Mesa (2005) que se vieron obligados a renunciar antes de finalizar su periodo presidencial. Una de las principales inconformidades de las poblaciones indígenas es la sensación de no pertenecía a sus respectivas naciones; la exclusión en la construcción y desarrollo político y social de sus Estados-nación; situación que surge desde la construcción del Estado-nación durante el siglo XIX.

\section{Identidad y cultura para la construcción de Estados-nación en América Latina}

En el constructo de nación en América Latina, la identidad y cultura se posicionan como dos de los temas centrales. Las nociones de identidad, multiculturalidad e idea de nación en las poblaciones indígenas es desarrollada por Chávez (1998b), quien enuncia que la identidad y representación de comunidades indígenas fue excluida en el proceso político durante la colonización. Sobre el territorio y la identidad, Castillo \& Cairo (2002a) y Mamani (2019b) convergen en que el territorio es un factor relevante en las identidades indígenas, hecho que, moldea las peticiones de libre determinación. Castillo \& Cair (2002a), señalan que los movimientos indígenas impulsan el Estado pluriétnico y multicultural, situación que, desafía el imaginario de nación mestiza. En Colombia, el multiculturalismo se vio representado por la inserción de fuerzas políticas indígenas en la arena electoral (Laurent, 2016). Por último, Stefanoni (2003a), menciona al nacionalismo indígena como identidad política que genera integración de movimientos, pueblos y comunidades indígenas.

Algunos autores abordan el tema de la identidad y la participación política de los pueblos indígenas y el concepto del Buen Vivir. En primer lugar, se encuentran los autores Espín-León \& Jimeno-Morenilla (2020), con su texto "Design of an instrument to measure indigenous cultural identity: Case study on the waorani amazonian nationality", en el que expresan que debido al impacto de las culturas occidentales los pueblos indígenas han sufrido cambios en su identidad cultural, específicamente en la Amazonía ecuatoriana, por lo que deciden crear un instrumento que determine el grado de los cambios en la identidad. Los resultados del instrumento mostraron que sí hay diferencias entre la identidad cultural de los indígenas, específicamente en el caso de la nacionalidad Waorani, con el resto de las poblaciones a las que se les aplicó el instrumento.

El libro "La Identidad Nacional", de Anthony Smith (1997), afirma que las personas tienen múltiples identidades y roles que son construidos por el entorno, la cultura, el territorio, así como por el género, la clase social, la religión, entre otros; de tal manera que la identidad tiene una función interna y externa en el ser humano. Por último,

39 Estabilidad política: "La estabilidad política y la ausencia de violencia. Ello refiere a la probabilidad de que el gobierno no sea desestabilizado o derrocado por medios violentos o inconstitucionales" (Organización de Estados Americanos, n.d.) 
Kloosterman (1997), en su libro "Identidad Indígena. Entre romanticismo y realidad", evidencia, a través de un trabajo de campo que realizó en un resguardo indígena en Colombia, que los indígenas tienen problemas con el reconocimiento de sus derechos colectivos, como el derecho a la tierra y a la autonomía; por ende, tienen problemas con su identidad.

Por otro lado, los movimientos indígenas hicieron parte de la fundamentación teórica y política del Estado plurinacional de Ecuador, el cual tenía críticas por la idea de nación que se había empleado pues, a pesar de ser una República, se mantenían formas de explotación y exclusión similar al periodo colonial (Fernández, 2018). Ecuador también incursionó el concepto del Buen Vivir en su Constitución, sin embargo, los autores Caria, Domínguez \& León (2017) argumentan que se trata de una tradición inventada que no representa por completo la cosmovisión de los pueblos indígenas.

\section{Regionalismo indígena, pasado común e identidad nacional}

Otros autores abarcan los temas de identidad, frontera, sistema jurídico, cultura indígena, nación, Estado y regionalismo indígena como convergentes. Con respecto a la identidad, el artículo "From identity to pluri-identity: The reworking of identity among Peruvian Pentecostal migrants and indigenous Bolivians in Arica and Iquique" (Mansilla et al., 2018) sostienen que las identidades son dinámicas y que los pueblos indígenas tienen pluridentidades que se adecuan a su contexto, en especial cuando son migrantes. Las identidades son construcciones sociales que se relacionan con lo nacional, la religión, el género, su condición migrante, entre otros aspectos. En relación con las fronteras, Minerva Campion (2018) dice que estas son construidas desde las poblaciones indígenas, pues son ellas quienes les dan significado a estas zonas fronterizas, contribuyen en sus procesos y mantienen las actividades culturales.

Natividad Gutiérrez (2018), en su artículo "Indigenous myths and nation building in Latin America”, expresa que el pasado étnico indígena es esencial para construir la identidad, y que los mitos también son creadores de identidad y nación. Sin embargo, concluye que los mitos no cumplen con esa función, sino que son las escuelas públicas las que forman a los ciudadanos nacionales. Macrena del Pilar Manzanelli (2020) habla sobre las experiencias identitarias de los pueblos indígenas Chuschagasta y Tolombón ${ }^{40}$, y cómo estas están marcadas por la imposición de la identidad nacional blanca, que busca implantar una identidad homogénea y europea en todos sus habitantes. A pesar de la incidencia biopolítica, los pueblos desafían estas medidas y mantienen sus prácticas, formas de trabajo, vínculos y organizaciones colectivas.

Por otro lado, es difícil lograr una interacción fácil entre los indígenas y las entidades estatales. Este es el caso de Brasil, donde los funcionarios públicos no atienden de manera adecuada los derechos de los ciudadanos indígenas debido a la falta de comunicación y entendimiento entre ellos. A ello se suma la dificultad de reconocer su presencia en el territorio, pues se cree que hay un estereotipo de la persona indígena en la que todos los que se identifican como tal deben estar, y quienes no entran son considerados como falsos (Maher \& Cavalcanti, 2019). Por su parte, Chile ha integrado en su sistema legal prácticas y discursos de pueblos indígenas, como el Mapuche; a pesar

40 Pueblos indígenas ubicados en Argentina. 
de ello, predominan las practicas jurídicas tradicionales, dejando de lado aspectos de la cultura mapuche que deberían estar contemplados desde el inicio (Reyes \& Santis, 2016).

La cultura indígena es un aspecto fundamental que los Estados tienen en cuenta para integrar a esta población. El artículo "Hybrid houses and dispersed communities: negotiating governmentality and living well in peruvian Amazonia" (Killick, 2021), examina, desde la comunidad Nativa Ashaninka, cómo los indígenas responden a las expectativas y solicitudes de estar dentro del Estado de Perú. Una estrategia para implementar es proyectar y transmitir la cultura indígena, para lograr y mantener su supervivencia en la Amazonía actual. El autor Bartholomew Dean (2015) expresa que en Perú es necesario formular el entorno educativo, que es respaldado por el Estado, para integrar el conocimiento indígena y lograr la supervivencia de la cultura de los pueblos indígenas.

Gaya Makaran-Kubis (2009) presenta el debate intelectual que existe sobre el concepto de nación y la diferencia que hay entre los pueblos de Europa Central-Oriental y el pueblo Aymara. Este último promueve el etnonacionalismo ${ }^{41}$ como parte de su proyecto "indionacionalismo", en el que la construcción identitaria y territorial está determinada por la unión Aymara y de otros pueblos indígenas que tienen una misma raíz lingüística y cultural. Por otro lado, Roberta Rice (2021) habla del surgimiento de un regionalismo indígena en los Andes, específicamente en Bolivia y Ecuador, que contempla una integración regional basada en normas, necesidades y prioridades locales dadas por los esfuerzos de los movimientos indígenas en defensa de su identidad, su territorio y su autonomía.

\section{Reclamo de derechos territoriales, proyecto nación y libre determinación de los pueblos}

Los derechos que poseen y reclaman los pueblos indígenas es lo que más son desarrollados por autores como Penelope Anthias (2018) quien expresa que en el contexto de economías neoextractivistas, con el aumento de la extracción de recursos en territorios indígenas, sus habitantes piden el reconocimiento de sus derechos territoriales. Los pueblos indígenas manifiestan que estas actividades afectan negativamente su ecosistema, sus derechos territoriales e intensifica el reclamo de su soberanía. Para defender sus intereses y reclamar sus derechos ante los Estados y corporaciones multinacionales, las comunidades indígenas de América Latina han implementado las manifestaciones como una herramienta común y legítima que genera presión y les permite expresarse ante estas situaciones (Hanna et al., 2016).

El artículo "Conformación de gobiernos indígenas mediante normas y procedimientos propios en Bolivia a partir del reconocimiento del derecho a la libre determinación de los pueblos" contiene una recopilación literaria de la lucha histórica que han realizado los pueblos indígenas en Bolivia, por el reconocimiento del derecho a la libre determinación, lo que permite tener su propia forma de gobierno, eligiendo ellos mismos sus autoridades, normas y procedimientos (Mamani, 2019a). Así como se reconoce la lucha de los movimientos indígenas por sus derechos, autores como Margarita Chávez (1998)

41 Forma de nacionalismo en la que la idea de "nación" es definida por una etnia. 
también reconocen la segregación que han pasado los colonos en este contexto, con el establecimiento de, por ejemplo, los resguardos indígenas. A pesar de ello, los colonos han logrado integrarse a los procesos establecidos por los pueblos indígenas, a tal punto que, en el Putumayo ${ }^{42}$, un colono logró ser gobernador indígena del asentamiento Buenavista, identificándose a sí mismo como indígena.

La vida de las personas es afectada por la manera en que la política del historicismo ${ }^{43}$ determina el discurso jurídico. Ello se debe a que se genera un régimen que organiza por fases la vida de las personas, como si siguiera un orden y secuencia determinada. Sin embargo, la vida de las personas, especialmente de los pueblos indígenas, no sigue esa forma de organización lineal del tiempo, sino que tiene distintos sentidos y formas que no se puede determinar desde el inicio (López \& Rojas, 2020). En este orden, Christian Gros (2002) en su artículo "América Latina: ¿identidad o mestizaje? La nación en juego" analiza la historicidad ${ }^{44}$ de los pueblos indígenas, para determinar el lugar que ellos han tenido en el proyecto nacional de los países de América Latina. Para lograr las naciones pluriétnicas y multiculturales que consagran las nuevas constituciones, el autor expresa que aún falta memoria y olvido por parte de las mismas naciones.

Sergio Solano (2012) expresa que durante el sigloXIX el Estado influyó en la construcción de la identidad indígena. En este contexto, los pueblos indígenas aprovecharon los conflictos políticos entre los sectores dirigentes y las normas republicanas para defender su posición y el derecho sobre la tierra. Las comunidades indígenas también aprovecharon su identidad para que los Estados, entre ellos Colombia y Ecuador, sean reconocidos como pluriétnicos y multiculturales (Castillo \& Cairo Carou, 2002b). Al ser contemplados como sujetos políticos, y al concebir la identidad y el territorio con un arma de lucha, los indígenas desafiaron el constructo de Estado-nación unitario y reinventaron la identidad étnica.

También se ha dado una participación política por parte de los pueblos indígenas. El artículo "El nacionalismo indígena como identidad política: la emergencia del MAS-IPSP (1995-2003)" muestra el ciclo de movilizaciones que se dieron en Bolivia a inicios del año 2000, como resultado del surgimiento de una conciencia nacional indígena y popular que trajo consigo el Movimiento al Socialismo-Instrumento Político por la Soberanía de los Pueblos (Stefanoni, 2003b). El documento "Elites and indigenousness in Colombia: Challenges of democracy in a context of multiculturalism" argumenta que el accionar indígena en la arena política, a inicio del siglo XIX, permitió la articulación entre las élites y los pueblos indígenas; lo que dio paso a la Constitución de 1991 y a la integración de los indígenas en la política (Laurent, 2016).

42 Departamento de Colombia, ubicado en el sur del país, que limita con los departamentos de Amazonas, Caquetá y Nariño, y con los países Ecuador y Perú.

43 Los autores López \& Rojas (2020) expresan que el historicismo "construye una idea de tiempo lineal, progresivo y ascendente". Por medio de esta se "crea una historia única mediante la organización de la multiplicidad de los eventos globales dentro de una narrativa peculiar organizada por la progresión del principio de la razón humana, la racionalidad técnica y el poder sobre la naturaleza".

44 Acontecimientos o hechos de importancia y trascendencia que forman parte de la historia. 


\section{Movilizaciones, reconocimiento y plurinacionalidad}

Los autores Rodolfo Stavenhagen (2002) y Geidy Morfa-Hernández (2020) afirman que los pueblos indígenas han sido invisibilizados en la construcción del Estado-nación en América Latina; por lo que ellos se han organizado para mantener su identidad y raíces, y reclamar sus derechos, luego de que se diera un proceso de imposición de la élite nacional sobre los pueblos indígenas. De esta manera, los pueblos indígenas han desafiado el concepto dominante de Estado-nación, al exigir el reconocimiento, garantía y respeto de sus derechos. El artículo de Nicolás Restrepo (2006), "The Catholic Church and the Colombian State; Combined Construction of a Nationality in the South of the Country", permite conocer el controvertido contexto en el que se dio el proceso de inserción de las sociedades indígenas del amazonas a la sociedad nacional en el siglo XIX. La iglesia católica fue la encargada de este proceso, y evidenció las diferencias entre las sociedades, las cuales se mantuvieron durante y después de este contexto.

Con las nuevas constituciones de los países latinoamericanos se buscó crear y mejora la relación existente con los pueblos indígenas; sin embargo, aun cuando estos países establecen ser plurinacionales, en la práctica terminando siendo autoritarios (MorfaHernández, 2020; Stavenhagen, 2002). Las únicas constituciones de América Latina que consagran los principios y derechos del Buen Vivir y la naturaleza con las de Ecuador y Bolivia. Empero, el artículo "El constitucionalismo radical ambiental y la diversidad cultural en América Latina. Los derechos de la naturaleza y el buen vivir en Ecuador y Bolivia" argumenta que no es un aporte original, sino que son reinterpretaciones de los conceptos de nación, pueblo y cultura, se da un uso y transformación de los conceptos (Bonilla Maldonado, 2018).

Karla Escobar (2020) afirma que dentro del proceso de formación del Estado-nación colombiano, las poblaciones indígenas del Cauca tenían distintas acciones para participar. La mayoría de estas eran plurales, diversas y en entornos de violencia, y una de las más usadas para reclamar sus derechos en el siglo XX eran las revueltas. Paola Vargas Moreno (2019) expresa en su artículo "Estrategias en disputa: educación superior y poblaciones indígenas en la construcción de los Estados-nación latinoamericanos" que la educación superior también contribuye al proyecto de Estado-nación, pero los pueblos indígenas han sido excluidos de su diseño. Por lo que ellos han recurrido a las movilizaciones, para construir de manera intercultural una educación superior que los visibilice. De igual forma, Enrique Mases (2010) habla de los relatos como contribuyentes en la formación del Estado-nación, al ser un eje de identificación de la identidad nacional. En Argentina hay un relato oficial de la historia que conoce toda la población, pues hace parte del aprendizaje histórico y formación académica; no obstante, los pueblos originarios siguen luchando por estar integrados y ser visibilizados en esa historia nacional.

En ese orden, el artículo "La disputa por el territorio durante la conformación del estado nacional y provincial. La batalla de quera y la demonización de los pueblos originarios" establece que en la construcción del Estado-nación, del proyecto de nacionalización e identidad nacional en Argentina, se conformó por acciones que buscaron deslegitimar los reclamos sobre territorios ancestrales que realizaban los pueblos indígenas. Estas acciones eran represivas y bélicas, hubo genocidios de pueblos indígenas, y todo hizo parte de la "Campaña de la puna" realizada por los terratenientes argentinos 
(Lenton et al., 2016). Los indígenas ubicados en la frontera colombo-ecuatoriana, específicamente en el departamento de Nariño-Colombia, también exigían el dominio sobre sus territorios ancestrales y buscaban la reconstrucción de su identidad perdida en las fronteras, como lo manifiesta Claudia Carrión (2015). Para ello, adelantaron procesos de autonomía territorial, formas organizativas y estructurales dentro del Estado colombiano, que les permitiera cumplir con su objetivo identitario. Stephen Baines (2012) habla de la frontera entre Brasil y Guayana, analiza las dinámicas fronterizas y las identidades nacionales de cada población. En la zona fronteriza las identidades coexisten sin problema, pues hay una relación histórica desde antes de que se establecieran las fronteras que aún se mantienen. La identidad no es exclusiva para los indígenas que habitan en esta zona, por lo que se identifican como nacionales brasileros y guyaneses al tiempo, aun cuando las respectivas autoridades de cada Estado tienden a manipular su identidad.

La pérdida de identidad cultural de las comunidades indígenas del Amazonas se refleja en el proceso de aculturación ${ }^{45}$ por el que ellas están pasando. El artículo "Cultural identity distance computation through artificial intelligence as an analysis tool of the amazon indigenous people. A case study in the waorani community" plantea un método para medir la distancia cultural entre individuos o grupos de individuos a través de técnicas de inteligencia artificial, puesto que es difícil medir la pérdida de identidad cultural. El documento concluye que la identidad cultural del pueblo indígena Waorani Amazónico está en proceso de aculturación debido a los flujos migratorios de poblaciones occidentales que transitan constantemente. En el caso de este pueblo, son las mujeres quienes mantienen la representación cultural, puesto que se relacionan menos con otras culturas externas al estar más en su territorio cuidando de sus hijos, conservando así su identidad indígena (Espín-León et al., 2020). La cosmovisión y cultura de los pueblos indígenas, como el Secoya ${ }^{46}$, muestran que hay una ontología del territorio diferente a la concebida por el Estado. Este último tiene una visión política y geográfica, mientras que el pueblo Secoya lo entiende desde su relación con la naturaleza y sus rituales, como lo indican Julián García Labrador \& José Ochoa (2020).

Con respecto a la nación, se detecta un problema con su concepción. Los autores Sofía Soria (2011), Bartolomé Clavero Salvador (2011), Claudia Gotta, Julio Risso y María Taruselli (2010) expresan que las narrativas de nación se reinventan en su relación con los otros: los pueblos indígenas, y que hay un lugar reservado para ellos a pesar de que se mantenga una concepción de nación homogenizada. Continúa un trato de exclusión con los pueblos indígenas que los aleja de la nación. En el caso colombiano la nación es fallida, pues la ciudadanía no se siente identificada en esta, aun cuando la constitución les da un reconocimiento a nivel nacional y también cuentan con reconocimiento internacional. Los autores concluyen que hace falta mejorar el trato que se les otorga a los indígenas, partiendo desde la educación, en la forma de concebir y hablar de los otros como parte central de la nación, y que se destruyan las fronteras que se han creado y mantenido hacia ellos.

45 La aculturación es un proceso en cual una cultura se adapta al contacto con otra cultura, exponiéndose a abandonar o perder su cultura propia, sin que eso suceda necesariamente.

46 Los Secoya se encuentran en la amazonia del Ecuador y de Perú. 
La historia es una herramienta implementada por los pueblos indígenas para mantener y transmitir su identidad y costumbres. La raza y la nacionalidad son construcciones sociales e históricas llenas de significado, y la historia del indigenismo y la nacionalidad muestran la importancia de asegurar el respeto y la protección legal de las diferentes formas de ser y pensar de cada persona (Devine, 2005). En el artículo "Construyendo el pasado territorial indígena. Comunalización y sentidos de pertenencia comechingón del Pueblo de La Toma (Ciudad de Córdoba)", la comunidad Comechingón fortalece la historia y su presencia en el territorio para demostrar que no se han extinguido. En este caso, es importante para la construcción de los procesos de comunalización ${ }^{47}$ contar con relatos provenientes de fuentes documentales y catastrales (Palladino, 2020). Carmen Martínez Novo (2014) expresa que, en Ecuador, los censos han sido una herramienta para (in) visibilizar los grupos minoritarios que están en el territorio, entre ellos los pueblos indígenas. Estos últimos sienten desconfianza del Estado cuando los registra y enumeran por raza o grupo étnica, pues la historia de los censos muestra que el Estado utiliza una estrategia de control social que minimiza el número de indígenas identificados y reconocidos en el país.

Por último, las acciones legales y los medios de comunicación les han permitido a los pueblos indígenas el reconocimiento y defensa de sus derechos. Los artículos "Partial recognition: rights of indigenous peoples in Chile" $\mathrm{y}$ "Beyond pluralism and media rights: indigenous communication for a decolonizing transformation of Latin America and Abya Yala" argumentan que los pueblos indígenas promueven sus objetivos de autonomía y autodeterminación a través de los medios de comunicación, desafiando la soberanía del Estado-nación. Las protestas que realiza el movimiento social indígena también han logrado políticas de reconocimiento que, según los autores, es débil; pues está condicionado por la defensa de la identidad que promueve el Estado-nación (Lehman, 2018; Fuentes \& De Cea, 2017).

\section{Narrativas, invisibilización y medios de comunicación}

En los artículos (i) "Indigenous cultural identity in the pedagogical discourse of history. A perspective of the Latin American curriculum", (ii) "The many lives of Lorenza Congo: narration and history in the Bolivian Amazon" $y$ (iii) "The public and private dimensions of a birthday party: politics, economics, and food in an Amazonian community" se aborda la identidad desde perspectivas diferentes. El primero, expresa que es necesario contar con un curriculum que contemple y enseñe la identidad y la interculturalidad en las escuelas de América Latina; para ir acorde con los discursos que promocionan los Estados sobre las identidades culturales indígenas (Turra Díaz et al., 2018). El segundo artículo muestra cómo una persona, en este caso Lorenza Congo, puede contemplarse como un símbolo de identidad indígena (Sturtevant, 2018). Sin embargo, la historia que se transmite de ella varía según la autoridad que la cuente, pues puede favorecer tanto a poblaciones, para posicionarse socialmente, y a gobiernos, para conseguir sus propios fines. El último documento expone que el cambio en las comidas y celebraciones tradicionales que realizan los pueblos indígenas surgen de condiciones

47 Lucas Palladino (2020) define los procesos de comunalización como el sentimiento que permite a los individuos estar juntos a partir de un grupo de acciones que les permite compartir y relacionarse. El autor expresa que: "En nuestro caso, se trata de la formación de comunidades aborígenes comechingonas que rescatan una historia y una identidad como comechingones y que encaran un proceso de reorganización territorial y política" 
políticas y económicas de la esfera pública y privada (Zycherman, 2016). En este caso, la celebración de la fiesta de cumpleaños por parte de los indígenas Tsimane, ubicados en la Amazonía boliviana, es una muestra de la integración de los pueblos indígenas marginados en los procesos estatales y en las economías monetarias.

Por otro lado, los autores Andreza de Souza Santos \& Sue Iamamoto (2019)feeding the Spanish and Portuguese empires with silver (Potosí, y Edviges Ioris (2018) manifiestan que las narrativas realizadas en América Latina, en especial durante la época colonial, eran racistas e invisibilizaban a los pueblos indígenas y africanos. En el siglo XXI los indígenas de Brasil realizaron contranarrativas y procesos étnicos y culturales para mostrar que seguían presentes en el territorio y exigir negociaciones de poder. Así como los pueblos indígenas y africanos eran explotados y empleados como mano de obra (de Souza Santos \& Iamamoto, 2019)feeding the Spanish and Portuguese empires with silver (Potosí, los territorios de la Amazonía también eran explotados por la hegemonía militar del gobierno civil de la Nueva República (1985-1990), debido a su riqueza natural y los beneficios mineros que generaba (Albert, 1992). El artículo "Autonomía Indígena Originario Campesina en las tierras altas de Bolivia" expresa que la ausencia de mecanismos efectivos para la defensa de los derechos a la autonomía y a la libre determinación en Bolivia, conlleva a que los pueblos indígenas no logren garantizar esos derechos. Por ello, la cooperación internacional se ha convertido en un actor relevante para el financiamiento de las estrategias de los movimientos indígenas en este país (Laurenti, 2017).

Los medios de comunicación de masa son usados por los pueblos indígenas para comunicar sus demandas sociales, influir en la toma de decisión y transmitir su cultura e identidad. El artículo "Indigenous Amazonians on air: Shipibo-Konibo radio broadcasters and their social influence in Peru" aborda el caso del pueblo peruano Shipibo-Konibo, que utiliza la radio local para hablar, por ejemplo, sobre el conflicto que tienen con la tala ilegal y la minería. En este sentido, Waldemar Henrique es considerado como un compositor regionalista que transmite en sus canciones, mitos y creencias la cultura popular amazónica, y contribuye a la construcción simbólica de nación (Aguiar \& De Miranda Clímaco, 2019). Tanto los radio-comunicadores como los compositores son un actor importante que transmiten y defienden lo que pasa en el Amazonas en su ámbito social, económico, político, cultural, ambiental, entre otros.

\section{Conclusiones}

Las poblaciones indígenas en América Latina no han apropiado el concepto de identidad nacional debido a que han sido excluidas en el diseñó del Estado-nación, pues ellos no fueron tenidos en cuenta en este proceso que buscó homogeneizar a la población sin reconocer la diversidad de identidades presentes en un mismo territorio. Durante las Repúblicas las poblaciones indígenas siguieron e, inclusive, fueron más explotadas que durante la época colonial. Asimismo, la idea de desarrollo social, económico y político planteado por los Estado-nación no ha logrado armonizarse y choca con la cosmovisión e identidad de los pueblos indígenas.

La identidad de los pueblos indígenas fue constituida siglos antes de la llegada de los colonizadores; esta gira en torno al hombre y su relación con la naturaleza. Durante el proceso de independencia de las colonias, los Estados latinoamericanos iniciaron 
su proyecto de nacionalización y construcción de Estado-nación; proceso en el cual, la identidad y cultura de las poblaciones indígenas fueron obviadas. Los Estados buscaban homogenizar a la población bajo una misma identidad, sin embargo, no se reconoció que dentro de un mismo territorio conviven diversas identidades; algunos ejemplos son Bolivia, Brasil, Colombia, Ecuador y Perú.

La educación fue una herramienta clave en el intento de homogenizar a la población y, por ello, la intervención de los pueblos indígenas en el sistema educativo es fundamental para luchar contra esa exclusión de las comunidades en el constructo de nación, así como el apoyo de actores internacionales. Las comunidades indígenas consideran que la exclusión se sigue manteniendo hoy en día, y para ello han recurrido a alternativas que les permitan expresar sus necesidades, mantener su identidad y ser escuchados tanto por las entidades nacionales como internacionales; estas alternativas se evidencian en la lucha por la libre determinación y las movilizaciones sociales.

\section{Referencias}

Aguiar, G. G., \& De Miranda Clímaco, M. (2019). Waldemar henrique a paraense composer: Regionalism and interaction with the nationalism of mário de andrade and getúlio vargas. Opus, 25(3), 281-307. https://doi.org/10.20504/ opus2019c2513

Albert, B. (1992). Indian Lands, Environmental Policy and Military Geopolitics in the Development of the Brazilian Amazon: The Case of the Yanomami. Development and Change, 23(1), 35-70. https://doi.org/10.1111/j.1467-7660.1992.tb00438.x

Anthias, P. (2018). Indigenous Peoples and the New Extraction: From Territorial Rights to Hydrocarbon Citizenship in the Bolivian Chaco. Latin American Perspectives, 45(5), 136-153. https://doi.org/10.1177/0094582X16678804

Baines, S. G. (2012). O movimento político indígena em Roraima: identidades indígenas e nacionais na fronteira Brasil-Guiana. Caderno CRH, 25(64), 33-44. https://doi.org/10.1590/s0103-49792012000100003

Bonilla Maldonado, D. (2018). El constitucionalismo radical ambiental y la diversidad cultural en América Latina. Los derechos de la naturaleza y el buen vivir en Ecuador y Bolivia. Revista Derecho Del Estado, 42, 3-23. https://doi. org/10.18601/01229893.n42.01

Campion, M. (2018). The Construction of the Amazonian Borderlands through the longue durée: An Indigenous Perspective. Journal of Borderlands Studies, 33(1), 123-140. https://doi.org/10.1080/08865655.2016.1226926

Carrión, C. P. (2015). Desafiando al Estado-nación: Reclamos de autonomía del pueblo pasto. Universitas Humanística, 80(80). https://doi.org/10.11144/javeriana. uh80.denr

Castillo, L. C., \& Cairo Carou, H. (2002a). Reinvención de la identidad étnica, nuevas 
territorialidades y redes globales: el Estado multiétnico y pluricultural.

Castillo, L. C., \& Cairo Carou, H. (2002b). Reinvención de la identidad étnica, nuevas territorialidades y redes globales: el Estado multiétnico y pluricultural. Revista Sociedad y Economía, 3, 55-76.

Chávez, M. (1998a). IDENTIDAD Y REPRESENTACIÓN ENTRE INDÍGENAS Y COLONOS DE LA AMAZONIA OCCIDENTAL COLOMBIANA. In Identidad, modernidad y desarrollo (pp. 283-296).

Chávez, M. (1998b). IDENTIDAD Y REPRESENTACIÓN ENTRE INDÍGENAS Y COLONOS DE LA AMAZONIA OCCIDENTAL COLOMBIANA 1 Margarita Chaves Chamorro Investigadora ICANH. 283-296.

Clavero Salvador, B. (2011). Nación y naciones en Colombia entre constitución, concordato y un convenio (1810-2010). Revista de Historia Del Derecho, 41, 79137. http://www.bicentenario.gov.co

Dávalos, P. (2005). Movimientos indígenas en América Latina: el derecho a la palabra. Pueblos Indígenas, Estado y Democracia, 17-33. http://bibliotecavirtual.clacso.org. ar/clacso/gt/20101026124338/2Davalos.pdf

de Souza Santos, A. A., \& Iamamoto, S. A. S. (2019). The Difficult Legacy of Mining in Past and Contemporary Potosí and Ouro Preto. Journal of Latin American Geography, 18(3), 110-131. https://doi.org/10.1353/lag.2019.0054

Dean, B. (2015). Identity and indigenous education in peruvian amazonia. In Indigenous Education: Language, Culture and Identity (pp. 429-446). Springer Netherlands. https://doi.org/10.1007/978-94-017-9355-1_21 PMid:26044338 PMCid:PMC4561433

Devine, T. (2005). “Diacuî" killed Iracema': Indigenism, nationalism and the struggle for Brazilianness. Bulletin of Latin American Research, 24(1), 92-122. https://doi. org/10.1111/j.0261-3050.2005.00126.x

Domínguez, R., Caria, S., \& León, M. (2017). Buen Vivir: Praise, instrumentalization, and reproductive pathways of good living in Ecuador. Latin American and Caribbean Ethnic Studies, 12(2), 133-154. https://doi.org/10.1080/17442222.2 017.1325099

Escobar, K. (2020). ¿Qué significa ser ciudadano e 'indio'?: sobre la diversidad de formas de apelar a la ciudadanía indígena en el Cauca (Colombia), 1902-1939. Latin American and Caribbean Ethnic Studies, 00(00), 1-24. https://doi.org/10.10 80/17442222.2020.1770974

Espín-León, A. M., Jimeno-Morenilla, A., Pertegal-Felices, M. L., \& Azorín-López, J. (2020). Cultural identity distance computation through artificial intelligence as an analysis tool of the amazon indigenous people. A case study in the waorani community. Sustainability (Switzerland), 12(22), 1-16. https://doi.org/10.3390/ su12229513 
Fernández, B. S. (2018). Indigenous Intellectuals in Contemporary Ecuador: Encounters with "Seven Erroneous Theses about Latin America." Latin American Perspectives, 45(2), 190-204. https://doi.org/10.1177/0094582X17747314

Fuentes, C., \& De Cea, M. (2017). Reconocimiento débil: Derechos de pueblos indígenas en Chile. Perfiles Latinoamericanos, 25(49), 55-75. https://doi.org/10.18504/ pl2549-003-2017

García Labrador, J., \& Ochoa, J. (2020). Two ontologies of territory and a legal claim in the ecuadorian upper amazo. Journal of Political Ecology, 27(1), 496-516. https:// doi.org/10.2458/V27I1.23099

Gotta, C. A., Risso, J. L., \& Taruselli, M. V. (2010). Repensar el Bicentenario de la margi-nación: Ciudadanía y pueblos indígenas. Revista Pilquen. Sección Ciencias Sociales, 12(12), 1-10.

Gros, C. (2002). América Latina: ¿identidad o mestizaje? La nación en juego. 127-147.

Gutierrez, N. (2018). Indigenous myths and nation building in Latin America. Nations and Nationalism, 24(2), 271-280. https://doi.org/10.1111/nana.12387

Hanna, P., Langdon, E. J., \& Vanclay, F. (2016). Indigenous rights, performativity and protest. Land Use Policy, 50, 490-506. https://doi.org/10.1016/j. landusepol.2015.06.034

Ioris, E. M. (2018). Memory regimes, struggles over resources and ethnogenesis in the brazilian amazon. Vibrant Virtual Brazilian Anthropology, 15(2), 1-23. https:// doi.org/10.1590/1809-43412018v15n2a405

Killick, E. (2021). Hybrid houses and dispersed communities: Negotiating governmentality and living well in Peruvian Amazonia. Geoforum, 119(August 2019), 279-288. https://doi.org/10.1016/j.geoforum.2019.08.003

Kloosterman, J. (1997). Identidad indígena, 'entre romanticismo y realidad' : el derecho a la autodeterminación y la tierra en el resguardo Muellamués en el sur-oeste de Colombia. Centro Cultural Abya Yala Del Ecuador. https://digitalrepository.unm. edu/abya_yala/539

Laurent, V. (2016). Elites and indigenousness in Colombia: Challenges of democracy in a context of multiculturalism. Elites and Indigenousness in Colombia: Challenges of Democracy in a Context of Multiculturalism, 87(87), 145-169. https://doi. org/10.7440/colombiaint87.2016.06

Laurenti, D. (2017). Autonomía Indígena Originario Campesina en las tierras altas de Bolivia. Izquierdas, 36, 222-252. https://doi.org/10.4067/S071850492017000500222 
Lehman, K. (2018). Beyond Pluralism and Media Rights: Indigenous Communication for a Decolonizing Transformation of Latin America and Abya Yala. Latin American Perspectives, 45(3), 171-192. https://doi.org/10.1177/0094582X18766911

Lenton, D. I., Piaggi, L. A., Seldes, V., Del, I., \& Salas, V. (2016). La disputa por el territorio durante la conformación del estado nacional y provincial. La batalla de quera y la demonización de los pueblos originarios. Cuadernos de La Facultad de Humanidades y Ciencias Sociales , 49, 271-292.

López, O. A., \& Rojas, T. E. (2020). La Politica del Historicismo en el Discurso Jurídico Multicultural: la Jurisprudencia Constitucional sobre la Identidad de los Pueblos Indígenas y la Idea de Nación en Colombia. Seqüência: Estudos Jurídicos e Políticos, 43(84), 10-35. https://doi.org/10.5007/2177-7055.2020v43n84p10

Maher, T. M., \& Cavalcanti, M. do C. (2019). Unseen and unheard: cultural identities and the communicative repertoires of Indios in Brazilian cities. Current Issues in Language Planning, 20(1), 50-66. https://doi.org/10.1080/14664208.2018.150 3387

Makaran-Kubis, G. (2009). El nacionalismo étnico en los Andes. El caso de los aymaras bolivianos. Latinoamérica. Revista de Estudios Latinoamericanos, 49, 35. https:// doi.org/10.22201/cialc.24486914e.2009.49.57425

Mamani, I. (2019a). Conformación de gobiernos indígenas mediante normas y procedimientos propios en Bolivia a partir del reconocimiento del derecho a la libre determinación de los pueblos. Revista Jurídica Derecho, 8.

Mamani, I. (2019b). MEDIANTE NORMAS Y PROCEDIMIENTOS PROPIOS PUEBLOS.

Mansilla, M. Á., Piñones, C., \& Liberona, N. (2018). De la identidad a la pluridentidad. Reelaboraciones identitarias entre pentecostales migrantes peruanos y bolivianos indígenas en Arica e Iquique. Si Somos Americanos, 18(2), 10-33. https://doi. org/10.4067/s0719-09482018000200010

Manzanelli, M. del P. (2020). «Somos pueblo, con autodeterminación libre y colectiva» reivindicaciones identitarias-organizativas de Los Chuschagasta y Tolombón. Tabula Rasa, 1(34), 109-130. https://doi.org/10.25058/20112742.n34.06

Martínez, C. (2014). The Minimization of Indigenous Numbers and the Fragmentation of Civil Society in the 2010 Census in Ecuador. Journal of Iberian and Latin American Research, 20(3), 399-422. https://doi.org/10.1080/13260219.2014.9 95877

Mases, P. E. (2010). La Construcción Interesada De La Memoria Histórica : the Interested Construction of the Historical Memory : the Myth of White Nation and the Invisibility of the Aborigine Community. 1-9. 
Morfa-Hernández, G. (2020). ¿Estado-nación o Estado plural? Pueblos indígenas y el Estado en América Latina (siglo XXI). Libro coordinado por Gaya Makaran. Estudios Sociológicos deEl Colegio de México, 38(114),pp.https://doi.org/10.24201/ es.2020v38n114.1836

Organización de Estados Americanos. (n.d.). 5. CAPíTULO IV - INFORME SOBRE EL ACCESOALAINFORMACIÓNENEL HEMISFERIO. RetrievedSeptember 16, 2021, from http://www.oas.org/es/cidh/expresion/showarticle.asp?artID=437\&lID=2

Palladino, L. (2020). Construyendo el pasado territorial indígena. Comunalización y sentidos de pertenencia comechingón del Pueblo de La Toma (Ciudad de Córdoba). Revista de Geografía Norte Grande, 102(75), 83-102. https://doi. org/10.4067/s0718-34022020000100083

Pertegal-Felices, M. L., Espín-León, A., \& Jimeno-Morenilla, A. (2020). Diseño de un instrumento para medir identidad cultural indígena: caso de estudio sobre la nacionalidad amazónica Waorani. Revista de Estudios Sociales, 75(71), 51-67. https://doi.org/10.7440/res71.2020.05

Restrepo, N. (2006). The Catholic Church and the Colombian State; Combined Construction of a Nationality in the South of the Country. Tabula Rasa, 1(5), 151-166. https://doi.org/10.25058/20112742.272

Reyes, A. A., \& Santis, F. J. (2016). Antropología jurídica y superposición de sistemas normativos estado/nación-pueblos indígenas: El caso actual del pueblo Mapuche. AIBR Revista de Antropologia Iberoamericana, 11(3), 319-340. https://doi. org/10.11156/aibr.110302

Rice, R. (2021). Indigenous regionalism in the Andes. Globalizations. https://doi.org/1 $0.1080 / 14747731.2021 .1907734$

Smith, A. (1997). La Identidad Nacional Y Otras Identidades. In La Identidad Nacional (pp. 1-16). Trama Editorial. http://historia.ihnca.edu.ni/ccss/dmdocuments/ Bibliografia/CCSS2009/Tema1/1/La_ident_nacional.pdf

Solano, S. P. (2012). Tributo, fenotipo y genealogía. Indígenas y nación en el Caribe Colombiano durante el siglo XIX. Revista Complutense de Historia de America, 38, 103-127. https://doi.org/10.5209/rev_RCHA.2012.v38.40236

Soria, S. (2011). La reinvención de la nación en la Argentina actual: estado, relato nacional y pueblos indígenas. Nómadas, 215-227. http://www.scielo.org.co/ scielo.php?script=sci_abstract\&pid=S0121-75502011000100015\&lng=en\&nr $\mathrm{m}=\mathrm{iso} \& \mathrm{tlng}=\mathrm{es}$

Stavenhagen, R. (2002). Identidad indígena y multiculturalidad en america latina.

Stefanoni, P. (2003a). El nacionalismo indígena como identidad política: La emergencia del MAS-IPSP (1995-2003). 2004, 1-49.

Stefanoni, P. (2003b). El nacionalismo indígena como identidad política: La emergencia del MAS-IPSP (1995-2003). 1-49. 
Sturtevant, C. (2018). Las múltiples vidas de Lorenza Congo: narración e historia en la Amazonia boliviana. Revista Complutense de Historia de América, 44, 67-88. https://doi.org/10.5209/rcha.61080

Turra Díaz, O., Lagos Pando, M., \& Valdés Vera, M. (2018). Identidad Cultural Indígena En El Discurso Pedagógico De La Historia. Una Mirada Al Currículum Latinoamericano. Diálogo Andino, 57, 49-60. https://doi.org/10.4067/s071926812018000300049

Vargas Moreno, P. A. (2019). Estrategias en disputa: educación superior y poblaciones indígenas en la construcción de los Estados-nación latinoamericanos. Cultura y Representaciones Sociales, 13(26), 97-128. https://doi.org/10.28965/2019-26-05

Zycherman, A. (2016). The public and private dimensions of a birthday party: Politics, economics, and food in an Amazonian community. Food and Foodways, 24(3-4), 153-172. https://doi.org/10.1080/07409710.2016.1210889 